\title{
Space-times Containing Perfect Fluids and Having a Vanishing Conformal Divergence*
}

\author{
L. C. Shepley and A. H. Taub \\ University of California, Berkeley, California
}

Received March 6, 1967

\begin{abstract}
The solutions of the Einstein field equations are studied under the assumptions that (I) the source of the gravitational field is a perfect fluid, (2) the divergence of the conformal (Weyl) tensor vanishes, and $(3 \mathrm{a})$ either an equation of state exists such that $p=p(w), p$ being the pressure and $w$ the rest energy density, or $(3 \mathrm{~b})$ the rest particle density is conserved. Under assumptions (1), (2), and (3a) it is shown that the space-time is conformally flat and the metric is a RobertsonWalker metric. The flow is irrotational, shear-free, and geodesic. Under assumptions $(1),(2)$, and $(3 \mathrm{~b})$ it is shown that either the line element is static or the fluid has a very special caloric equation of state. Conditions for a static solution to exist are examined, and it is shown that the Schwarzschild interior solution satisfies these conditions as does the Einstein universe. The Schwarzschild interior and the Einstein universe are the only conformally flat, static solutions obeying (1), (2), and (3b).
\end{abstract}

\section{Introduction}

In this paper we shall discuss the space-times satisfying the Einstein field equations when the source of the gravitational field is a perfect fluid and which are such that the divergence of the conformal (Weyl) tensor vanishes.

It is a consequence of the latter condition that the four-velocity of the fluid has vanishing rotation and shear. In addition, the derivatives of the rest energy density $w$ in directions orthogonal to the four-velocity vanish. If the fluid obeys an equation of state, that is, if the pressure is a function of $w$ alone, it then follows that the particle paths of the fluid are geodesics. Thus the assumptions that the divergence of the conformal tensor vanishes and that an equation of state holds imply that the flow is geodesic, irrotational and shear free [1]. As is known, these conditions in turn imply that the space-time is conformally flat, and that the metric is of the Robertson-Walker type [2].

If the assumption that an equation of state holds is replaced by the assumption that the particle density is conserved, it follows that either the rest energy density of the fluid is constant, or the entropy is constant,

* The research reported herein was supported in part by the Atomic Energy Commission under contract number AT (11-1)-34, Project Agreement No. 125. 
or the fluid satisfies very special thermodynamic relations. In case the entropy is constant, an equation of state holds, and the metric is a Robertson-Walker metric. In case the rest energy density is constant, the metric is a static one, and the three-space orthogonal to the fourvelocity is a restricted one. If the energy density is constant and the space-time is conformally flat, then the metric tensor is that of the Schwarzschild interior solution or of the Einstein universe.

We shall adopt the convention that the metric tensor $g_{\alpha \beta}$ $(\alpha, \beta=0,1,2,3)$ have the signature $(-+++)$. The Riemann tensor will have its sign determined so that the Ricci relation becomes

where we use the notation

$$
2 v_{\alpha ;[\beta \gamma]}=v_{\sigma} R_{\alpha \beta \gamma}^{\sigma}
$$

$$
f_{[\alpha \beta]}=\frac{1}{2}\left(f_{\alpha \beta}-f_{\beta \alpha}\right)
$$

and

$$
f_{(\alpha \beta)}=\frac{1}{2}\left(f_{\alpha \beta}+f_{\beta \alpha}\right) .
$$

We define the Ricci tensor as

$$
R_{\mu \nu}=R_{\mu \sigma \nu}^{\sigma}
$$

and then the Einstein field equations become

$$
R_{\mu \nu}-\frac{1}{2} g_{\mu \nu} R=k T_{\mu \nu}
$$

where $k$ is Einstein's gravitational constant. The stress-energy tensor is assumed to be that of a perfect fluid and hence

with

$$
\begin{aligned}
T_{\mu \nu} & =[\varrho(1+\varepsilon)+p] u_{\mu} u_{v}+p g_{\mu \nu} \\
& =(w+p) u_{\mu} u_{\nu}+p g_{\mu \nu}
\end{aligned}
$$

$$
w=\varrho(1+\varepsilon)
$$

where $\varrho$ is the rest particle density, $p$ the pressure, and $\varepsilon$ the internal energy which is a function of $p$ and $\varrho$. For different fluids this function is different. The vector $u_{\mu}$ is the matter four-velocity vector which is a time-like unit vector and hence satisfies

$$
u^{\sigma} u_{\sigma}=-1 .
$$

We have omitted the cosmological constant $\Lambda$ in the field equations. It may be reintroduced by replacing $p$ by $p+\Lambda$ and $w$ by $w-\Lambda$.

As is well known the contracted Bianchi identities

$$
\left(R_{\alpha}^{\sigma}-\frac{1}{2} \delta_{\alpha}^{\sigma} R\right)_{; \sigma}=k T_{\alpha ; \sigma}^{\sigma}=0
$$


imply the equation of motion

where

$$
(w+p) A^{\alpha}=(w+p) u_{; \sigma}^{\alpha} u^{\sigma}=-p,{ }_{, \sigma} h^{\sigma \alpha}
$$

$$
h^{\sigma \alpha}=g^{\sigma \alpha}+u^{\sigma} u^{\alpha}
$$

and also imply the equation

$$
(w+p) \theta=(w+p) u_{; \sigma}^{\sigma}=-w_{, \sigma} u^{\sigma} .
$$

The vector $A^{\alpha}$ will be referred to as the acceleration vector, and the scalar $\theta$ will be called the dilitation.

In many physical problems Eq. (1.2), with the right hand side given by Eq. (1.3), are insufficient to determine the space-time and the motion of the matter in it. To obtain a complete description of the problem these equations must be supplemented by an additional equation. It is customary to use two forms of this additional restraint:

1. To require that the matter satisfy an equation of state. That is, to require that

or

$$
p=p(w)
$$

2. To require, as in classical hydrodynamics, that the rest particle density be conserved [3]. That is, to require that

$$
\left(\varrho u^{\sigma}\right)_{; \sigma}=0 \text {. }
$$

It follows from Eq. (1.4) that

$$
d w=d \varrho(1+\varepsilon)+\varrho d \varepsilon .
$$

We may express $d \varepsilon$ in terms of the temperature $T$ and the change in entropy $S$ by using the relation [3]

Hence

$$
T d S=d \varepsilon+p d\left(\frac{1}{\varrho}\right) .
$$

and Eq. (1.8) becomes

$$
d w=\frac{d \varrho}{\varrho}(w+p)+\varrho T d S
$$

$$
(w+p)\left(\varrho u^{\sigma}\right)_{; \sigma}+\varrho T S_{, \sigma} u^{\sigma}=0 .
$$

If particle rest mass is conserved, Eq. (1.8) is equivalent to

$$
S_{, \sigma} u^{\sigma}=0 \text {. }
$$

The rotation and shear associated with the fluid motion are defined as follows [4]: Write

where

$$
u_{\alpha ; \beta}=\omega_{\alpha \beta}+\sigma_{\alpha \beta}+\frac{1}{3} \theta h_{\alpha \beta}-A_{\alpha} u_{\beta}
$$

$$
\omega_{\alpha \beta}=u_{[\sigma ; \tau]} h_{\alpha}^{\sigma} h_{\beta}^{\tau}
$$


is called the rotation tensor, and

$$
\sigma_{\alpha \beta}=u_{(\sigma ; \tau)} h_{\alpha}^{\sigma} h_{\beta}^{\tau}-\frac{1}{3} \theta h_{\alpha \beta}
$$

is called the shear tensor.

The vorticity vector associated with the velocity vector $u^{\mu}$ is defined as [3]:

$$
v^{\mu}=(-g)^{-\frac{1}{2}} \varepsilon^{\mu \nu \sigma \tau} u_{\sigma ; \tau} u_{\nu}=(-g)^{-\frac{1}{2}} \varepsilon^{\mu \nu \sigma \tau} \omega_{\sigma \tau} u_{\nu}
$$

where $g$ is the determinant of the metric tensor and $\varepsilon^{\mu \nu \sigma \tau}$ is the completely antisymmetric tensor density with $\varepsilon^{0123}=1$. On multiplying this equation by $(-g)^{\frac{1}{2}} \varepsilon_{\mu \alpha \beta \gamma}$ and summing, we obtain

$$
(-g)^{\frac{1}{2}} v^{\mu} \varepsilon_{\mu \alpha \beta \gamma}=2 u_{\alpha} u_{[\beta ; \gamma]}+2 u_{\beta} u_{[\gamma ; \alpha]}+2 u_{\gamma} u_{[\alpha ; \beta]} .
$$

It then follows that

$$
\omega_{\beta \gamma}=-\frac{1}{2}(-g)^{\frac{1}{2}} v^{\mu} u^{\alpha} \varepsilon_{\mu \alpha \beta \gamma} .
$$

The vanishing of $v^{\mu}$ implies the vanishing of $\omega_{\beta \gamma}$ and conversely. Hence if $\omega_{\alpha \beta}=0, u_{\mu}$ is proportional to the gradient of a scalar.

The conformal (Weyl) tensor $C^{\alpha \beta_{\sigma \tau}}$ is defined in terms of the Riemann tensor, the Ricci tensor, and the scalar curvature $R$ by the equations [5]

$$
R^{\alpha \beta}{ }_{\sigma \tau}=C^{\alpha \beta}{ }_{\sigma \tau}+2 R^{\left[{ }_{\alpha}{ }_{[\sigma}{ }^{\beta j]}{ }_{\tau]}\right.}+\frac{1}{12} R \delta^{\alpha \beta}{ }_{\sigma \tau}
$$

where $\stackrel{\circ}{R}_{\beta}^{\alpha}$ is the traceless part of the Ricci tensor:

and as usual,

$$
\stackrel{\circ}{R}_{\beta}^{\alpha}=R^{\alpha}{ }_{\beta}-\frac{1}{4} \delta^{\alpha}{ }_{\beta} R
$$

$$
\delta^{\alpha \beta}{ }_{\sigma \tau}=\delta^{\alpha}{ }_{\sigma} \delta^{\beta}{ }_{\tau}-\delta^{\alpha}{ }_{\tau} \delta^{\beta}{ }_{\sigma} .
$$

The conformal (or Weyl) tensor is traceless on any contraction:

$$
C_{\beta \sigma}^{\alpha \sigma}=0 \text {. }
$$

The parts of the Riemann tensor are distinguished in the following manner [6]: Let us define the "hook" operator by

where

$$
R \cup_{\alpha \beta \gamma \delta}=\frac{1}{2} E_{\gamma \delta \sigma \tau} R_{\alpha \beta}^{\sigma \tau}, \quad \cup_{\alpha \beta \gamma \delta}=\frac{1}{2} E_{\alpha \beta \sigma \tau} R^{\sigma \tau}{ }_{\nu \delta}
$$

$$
E_{\alpha \beta \gamma \delta}=g^{\frac{1}{2}} \varepsilon_{\alpha \beta \gamma \delta} .
$$

Note that we are using $g^{\frac{1}{2}}$ and not $(-g)^{\frac{1}{2}}$, so that $E_{\alpha \beta \gamma \delta}$ is a pure imaginary tensor. Define $T^{\alpha \beta}{ }_{\sigma \tau}$ and $S^{\alpha \beta}{ }_{\sigma \tau}$ by

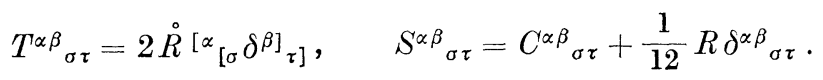


Then:

$$
\begin{aligned}
& \cup_{S \cup_{\alpha \beta \gamma \delta}}=S_{\alpha \beta \gamma \delta} \\
& \cup_{\alpha \beta \gamma \delta}=-T_{\alpha \beta \gamma \delta} .
\end{aligned}
$$

Hence $S_{\alpha \beta \gamma \delta}$ and $T_{\alpha \beta \gamma \delta}$ are, respectively, the self-"dual" and anti-self"dual" parts of $R_{\alpha \beta \gamma \delta}$ under the double hook operation. $C_{\alpha \beta \gamma \delta}$ is the traceless part of $S_{\alpha \beta \gamma \delta}$, and hence is distinguishable in an algebraic way from the $R$ term.

The three algebraically distinct parts of $R^{\alpha \beta \gamma \delta}$, as listed in Eq. (1.17), could be arbitrarily specified in relation to one another were it not for the fact that the Bianchi relations connect them differentially. This connection is expressed by noting that the Bianchi identities, which are

are equivalent to

$$
R_{\varrho \sigma ; \tau}^{\alpha \beta}+R_{\sigma \tau ; \varrho}^{\alpha \beta}+R^{\alpha \beta}{ }_{\tau_{\varrho} ; \sigma}=0
$$

$$
\cup_{R}^{\cup \alpha \beta \gamma \sigma} ; \sigma=0 \text {. }
$$

These equations may be written as

$$
C_{\gamma \delta ; \sigma}^{\alpha \sigma}-2\left(\stackrel{\circ}{R}_{[\gamma}^{\alpha}{ }_{[\gamma} \delta^{\sigma]}{ }_{\delta]}\right)_{; \sigma}+\frac{1}{12}\left(R \delta_{\gamma \delta}^{\alpha \sigma}\right)_{; \sigma}=0 .
$$

Hence we may write the Bianchi relations as [7]

$$
\left.C_{\gamma \delta ; \sigma}^{\alpha \sigma}=\left(R_{[\gamma}^{\alpha}-\frac{1}{6} R \delta_{[\gamma}^{\alpha}\right) ; \delta\right]
$$

which is simply Eq. (1.21) rewritten. It is this last form of the Bianchi equation which is most convenient when we insert the assumption that $C_{\gamma \delta ; \sigma}^{\alpha \sigma}=0$.

\section{Evaluation of the Conformal Divergence}

When the Ricci tensor and the scalar curvature are evaluated from the field equations, Eqs. (1.2) and (1.3), and the results substituted into Eq. (1.24) one obtains

$$
\left.k\left[(w+p) u^{\alpha} u_{[\gamma}+\frac{1}{3} w \delta^{\alpha}{ }_{[\gamma}\right] ; \delta\right]=C_{\gamma \delta ; \sigma}^{\alpha \sigma}
$$

Next, one may use Eqs. (1.6), (1.8), and (1.14) to write these equations as [7]

$$
\begin{gathered}
k^{-1} C_{\gamma \delta ; \sigma}^{\alpha \sigma}=(w+p) u^{\alpha} \omega_{\gamma \delta}+u^{\alpha} w,\left[\delta u_{\gamma]}+\frac{1}{3} w_{, \sigma} h^{\sigma}{ }_{[\delta} \delta_{\gamma]}^{\alpha}+\right. \\
+(w+p)\left(\omega^{\alpha}{ }_{[\delta}+\sigma^{\alpha}{ }_{[\delta}\right) u_{\gamma]} .
\end{gathered}
$$

Contract this equation with $u_{\alpha}$ and $u^{\gamma}$ to obtain

$$
\frac{1}{3} w,{ }_{, \sigma} h_{\delta}=k^{-1} u_{\alpha} u^{\gamma} C_{\gamma \delta ; \sigma}^{\alpha \sigma} \text {. }
$$


If Eq. (2.2) is contracted with $u^{\delta}$ we obtain

or

$$
k^{-1} C^{\alpha \sigma}{ }_{\gamma \delta \sigma} u^{\delta}=\frac{1}{3} u^{\alpha} w,{ }_{\sigma} h_{\gamma}^{\sigma}+\frac{1}{2}(w+p)\left(\omega_{\gamma}^{\alpha}+\sigma_{\gamma}^{\sigma}\right)
$$

$$
k^{-1} h_{\alpha \varrho} C^{\varrho \sigma}{ }_{\gamma \delta ; \sigma} u^{\delta}=\frac{1}{2}(w+p)\left(\omega_{\alpha \gamma}+\sigma_{\alpha \gamma}\right) .
$$

Hence we have

and

$$
\omega_{\alpha \beta}=2 k^{-1}(w+p)^{-1} u^{\varrho} h_{\sigma[\alpha} C_{\beta] \varrho ; \tau}^{\sigma \tau}
$$

$$
\sigma_{\alpha \beta}=2 k^{-1}(w+p)^{-1} u^{\varrho} h_{\sigma(\alpha} C_{\beta)_{\varrho} ; \tau}^{\sigma \tau} .
$$

Equations (2.3), (2.4), (1.6), and (1.8) contain all the information in the Bianchi identies. (Compare Szekeres [8].) It is evident that the necessary and sufficient condition for a space-time satisfying Eqs. (1.2) and (1.3) to have a conformal tensor with vanishing divergence is that the fluid motion be irrotational and shear-free and that

$$
w,{ }_{\sigma} h^{\sigma \alpha}=0
$$

hold. If, in addition, the fluid obeys an equation of state where $\frac{\partial p}{\partial w} \neq 0$, or if $p=$ constant, it follows from Eq. (1.6) that the fluid particles move along geodesics.

The two assumptions, vanishing divergence of the conformal tensor and the existence of an equation of state, imply that

$$
u_{\alpha ; \beta}=\frac{1}{3} \theta h_{\alpha \beta} .
$$

We shall show later that it is a consequence of this equation and the field equations that the metric tensor is that of a Robertson-Walker cosmological space $[9,2]$.

The vanishing of the divergence of the conformal tensor implies that

where

$$
u_{\alpha ; \beta}=\frac{1}{3} \theta h_{\alpha \beta}+F,{ }_{\tau} h_{\alpha}^{\tau} u_{\beta}
$$

and $\theta$ is given by Eq. (1.8).

$$
F=\log (w+p)
$$

We close this section with the derivation of two equations we shall find useful. The Bianchi identities, Eqs. (1.22), imply

$$
C^{\alpha \sigma}{ }_{\gamma \delta ; \sigma \varepsilon} E^{\gamma \delta \varepsilon \beta}=\left(R_{\gamma}^{\alpha}-\frac{1}{6} R \delta_{\gamma}^{\alpha}\right)_{; \delta \varepsilon} E^{\gamma \delta \varepsilon \beta}
$$

where $E^{\alpha \beta \gamma \delta}$ is given in Eq. (1.21). Make use of the Ricci identity and the hook operator of Eq. (1.20) to write Eq. (2.11) as

$$
-2 C^{\cup \alpha \sigma \beta \tau} ; \sigma \tau=\stackrel{\circ}{R}_{\sigma \tau} C^{\cup \alpha \sigma \beta \tau} \text {. }
$$

For a fluid-filled model, Eq. (2.12) becomes

$$
-2 k^{-1} C_{; \sigma \tau}^{\cup \alpha \sigma \beta \tau}=(w+p) u_{\sigma} u_{\tau} C^{\cup \alpha \sigma \beta \tau} \text {. }
$$


The second equation of interest will be an expression for $u_{\sigma} u_{\tau} C^{\alpha \sigma \beta \tau}$. To derive it, notice that for a perfect fluid

$$
u_{\sigma} u^{\tau} R_{\tau \beta}^{\sigma \alpha}=u_{\sigma} u^{\tau} C^{\sigma \alpha}{ }_{\tau \beta}+\frac{k}{6}(w+3 p) h^{\alpha}{ }_{\beta} .
$$

By the Ricci identity

$u_{\sigma} u^{\tau} R_{\tau \beta}^{\sigma \alpha}=\left(u_{; \tau \beta}^{\alpha}-u_{; \beta \tau}^{\alpha}\right) u^{\tau}=\left(u_{;}^{\alpha} u^{\tau}\right)_{; \beta}-u_{; \tau}^{\alpha} u_{; \beta}^{\tau}-u_{; \beta \tau}^{\alpha} u^{\tau}$.

Hence

$$
u_{\sigma} u^{\tau} C^{\sigma \alpha}{ }_{\tau \beta}+\frac{k}{6}(w+3 p) h_{\beta}^{\alpha}=\left(u_{; \tau}^{\alpha} u^{\tau}\right)_{; \beta}-u_{; \tau}^{\alpha} u_{; \beta}^{\tau}-u_{; \beta \tau}^{\alpha} u^{\tau} .
$$

We may substitute from Eq. (1.14) into these equations and thus express the right hand sides in terms of $\omega, \sigma, \theta, p$, and $w$. If in the resulting equation we set $\alpha=\beta$ and sum, we obtain an equation which reduces to the Raychaudhuri equation [10] when $p=0$. It is:

$$
\begin{aligned}
\frac{1}{2} k(w+3 p)= & u_{\sigma} u^{\tau} R_{\tau}^{\sigma}=-(w+p)^{-1}\left(p_{; \sigma \tau} h^{\sigma \tau}+p,_{\sigma} u^{\sigma} \theta\right)+ \\
& +(w+p)^{-2}\left(2 p_{, \sigma} p{ }_{,} h^{\sigma \tau}+p_{, \sigma} w_{, \tau} h^{\sigma \tau}\right)+ \\
& +\omega^{2}-\sigma^{2}-\frac{1}{3} \theta^{2}-\theta_{, \sigma} u^{\sigma}
\end{aligned}
$$

where $\omega^{2}=\omega^{\sigma \tau} \omega_{\sigma \tau}, \sigma^{2}=\sigma^{\sigma \tau} \sigma_{\sigma \tau}$.

When Eq. (2.17) is substituted into the expanded form of Eqs. (2.16) we obtain

$$
\begin{aligned}
u_{\sigma} u_{\tau} C^{\alpha \sigma \beta \tau}= & (w+p)^{-1}\left(-p_{; \sigma \tau} h^{\sigma \alpha} h^{\tau \beta}+\frac{1}{3} p_{; \sigma \tau} h^{\sigma \tau} h^{\alpha \beta}-p_{, \sigma} u^{\sigma} \sigma^{\alpha \beta}\right)+ \\
& +(w+p)^{-2}\left(p_{, \sigma} w{ }_{,} h^{\sigma}{ }^{\alpha} h^{\beta) \tau}-\frac{1}{3} p_{, \sigma} w{ }_{,} h^{\sigma \tau} h^{\alpha \beta}\right)+ \\
& +2(w+p)^{-2}\left(p_{, \sigma} p_{,} h^{\alpha \alpha} h^{\tau \beta}-\frac{1}{3} p_{, \sigma} p_{, \tau} h^{\sigma \tau} h^{\alpha \beta}\right)+ \\
& +\omega^{\alpha}{ }_{\tau} \omega^{\beta \tau}-\frac{1}{3} \omega^{2} h^{\alpha \beta}-\sigma^{\alpha}{ }_{\tau} \sigma^{\beta \tau}+ \\
& +\frac{1}{3} \sigma^{2} h^{\alpha \beta}-\frac{2}{3} \theta \sigma^{\alpha \beta}-h^{\alpha}{ }_{\varrho} h_{\sigma}^{\beta} \sigma^{\alpha \sigma}{ }_{; \tau} u^{\tau} .
\end{aligned}
$$

When the divergence of the conformal tensor vanishes, Eqs. (2.17) and $(2.18)$ reduce to

and

$$
\frac{1}{2} k(w+3 p)=-F_{\sigma \tau} h^{\sigma \tau}-\left[\frac{1}{3} \theta^{2}+\theta{ }_{, \tau} u^{\tau}+\theta F,{ }_{\tau} u^{\tau}\right]
$$

respectively, where

$$
u_{\sigma} u^{\tau} C^{\sigma \alpha}{ }_{\tau \beta}=-\left(F_{\sigma \tau} h^{\sigma \alpha} h_{\beta}^{\tau}-\frac{1}{3} F_{\sigma \tau} h^{\sigma \tau} h^{\alpha}{ }_{\beta}\right)
$$

$$
F_{\sigma \tau}=F_{; \sigma \tau}-F_{, \sigma} F_{, \tau}=(w+p)^{-1} p_{; \sigma \tau}-2(w+p)^{-2} p_{, \sigma} p_{, \tau}
$$

using $F$ as defined by Eq. (2.10). 
If, in addition, an equation of state obtains, then

and

$$
F,{ }_{\tau} h^{\tau \alpha}=0
$$

$$
\begin{aligned}
F_{\sigma \tau} h^{\sigma \alpha} h^{\tau}{ }_{\beta} & =-\frac{1}{3} \theta\left(u^{\sigma} F,_{\sigma}\right) h^{\alpha}{ }_{\beta} \\
F_{\sigma \tau} h^{\sigma \tau} & =-0\left(u^{\sigma} F,{ }_{\sigma}\right) .
\end{aligned}
$$

Equations (2.19) and (2.20) reduce to

and

$$
\frac{1}{2} k(w+3 p)=-\left(\frac{1}{3} \theta^{2}+\theta_{, \sigma} u^{\sigma}\right)
$$

respectively.

$$
C_{\beta}^{\alpha} \equiv u_{\sigma} u^{\tau} C^{\sigma \alpha}{ }_{\tau \beta}=0
$$

Thus the existence of an equation of state and the vanishing of the divergence of the conformal tensor imply that Eq. (2.22) holds and that

$$
\hat{C}_{\beta}^{\alpha} \equiv u_{\sigma} u^{\tau} C^{\cup \sigma \alpha}{ }_{\tau \beta}=0
$$

as follows from Eqs. (2.13). These two conditions in turn imply that

$$
C^{\sigma \alpha}{ }_{\tau \beta}=0 \text {. }
$$

That is, the space-time must be conformally flat. The proof that Eq. (2.24) is a consequence of Eqs. (2.22) and (2.23) rests on the following identity

$$
\begin{aligned}
\left(u_{Q} u^{\varrho}\right)^{2} C^{\alpha \beta}{ }_{\gamma \delta}= & \operatorname{Re}\left[u^{\sigma} u_{\tau}\left(\delta^{\alpha \beta}{ }_{\sigma \mu}+E^{\alpha \beta}{ }_{\sigma \mu}\right)\left(\delta^{\tau{ }^{v}}{ }_{\gamma \delta}+E^{\tau v}{ }_{\gamma \delta}\right)\left(C^{\mu}{ }_{\nu}+\hat{C}^{\mu}{ }_{\nu}\right)\right] \\
= & u^{\sigma} u_{\tau}\left[\delta^{\alpha \beta}{ }_{\sigma \mu} \delta^{\tau{ }{ }_{\gamma \delta}}+E^{\alpha \beta}{ }_{\sigma / l} E^{\tau v}{ }_{\gamma \delta}\right] C^{\prime \prime}{ }_{\nu}+ \\
& +u^{\sigma} u_{\tau}\left[E^{\alpha \beta}{ }_{\sigma \mu} \delta^{\tau v}{ }_{\gamma \delta}+\delta^{\alpha \beta}{ }_{\sigma \mu} E^{\tau v}{ }_{\gamma \delta}\right] \hat{C}^{\mu}{ }_{\nu}
\end{aligned}
$$

which holds when $u^{\varrho} u_{Q} \neq 0$ and is a consequence of the fact that the conformal tensor is self-dual under the double hook operation (compare HAWKING [1]).

\section{The Field Equations when an Equation of State Holds}

We now turn to a discussion of the field equations. We first discuss the situation when $S=$ constant or $p=p(w), \frac{\partial p}{\partial w} \neq 0$ and $w$ is not a constant throughout space-time. We shall of course assume that the divergence of the conformal tensor vanishes. We have already seen that these assumptions imply that the space-time is conformally flat and that the four-velocity vector is irrotational, shear-free, and geodesic. Hence, we have as a consequence of the geodesic character of the flow

and

$$
\omega_{\alpha \beta}=\frac{1}{2}\left(u_{\sigma ; \tau}-u_{\tau ; \sigma}\right) h_{\alpha}^{\sigma} h_{\beta}^{\tau}=\frac{1}{2}\left(u_{\alpha ; \beta}-u_{\beta ; \alpha}\right)=0
$$

$$
u_{\alpha ; \beta}=\frac{1}{3} \theta h_{\alpha \beta}=-\frac{1}{3}(w+p)^{-1} w_{, \sigma} u^{\sigma} h_{\alpha \beta} .
$$


We may therefore choose a function $t$ so that

$$
u_{\mu}=-t_{, \mu} .
$$

The function $t$ will be the zero'th coordinate function. Other coordinates $y^{i}, i=1,2,3$, may be chosen so that the metric components $g_{0 i}$ are all zero and $g_{00}=-1[5]$. The components of the matter velocity have the values

$$
u_{\alpha}=(-1,0,0,0) ; \quad u^{\alpha}=(1,0,0,0) .
$$

And as we mentioned, $w$ and $p$ are functions of $t$ only.

In these coordinates Eq. (3.1) reads

$$
(w+p) u_{i ; j}=-\frac{1}{3} \dot{w} g_{i j} \quad(i, j=1,2,3) .
$$

However, the derivatives of $u_{i}$ are given in terms of the Christoffel symbols by

Hence

$$
u_{i ; j}=\Gamma_{i j}^{0}=\frac{1}{2} g_{i j, 0} .
$$

$$
\left(g_{i j}\right)^{-1} \dot{g}_{i j}=-\frac{2}{3}(w+p)^{-1} \dot{w}=\text { func of } t \text { only }
$$

and the dot denotes the derivative with respect to $t=x^{0}$. Consequently,

$$
g_{i j}=\mathfrak{Q}^{2}(t) k_{i j}
$$

where the $k_{i j}$ are functions only of the spatial coordinates $y^{i}$.

The function $\mathfrak{R}(t)$ has been defined so that

$$
(w+p)^{-1} \dot{w}=-3 \Re-1 \dot{\gtrless} \text {. }
$$

This equation is equivalent to Eq. (1.8).

To find $k_{i j}\left(y^{k}\right)$ we must turn to the field equations. It is relatively straightforward to show that the spatial components of the Ricci tensor are

$$
R_{i j}=\mathfrak{R} \dddot{\Re} k_{i j}+2 \dot{\mathfrak{R}}^{2} k_{i j}+{ }^{3} R_{i j}
$$

where the quantity ${ }^{3} R_{i j}$ is the Ricci tensor formed from $k_{i j}$.

We make use of the field equations in the form

$$
R_{i j}=k\left(T_{i j}-\frac{1}{2} T_{\sigma}^{\sigma} \mathfrak{R}^{2} k_{i j}\right)=\frac{1}{2} k(w-p) \mathfrak{R}^{2} k_{i j}
$$

to show

$$
{ }^{3} R_{i j}=k_{i j}\left(-\mathfrak{R} \ddot{\mathfrak{R}}-2 \dot{\mathfrak{R}}^{2}+\frac{1}{2} k \mathfrak{R}^{2}(w-p)\right) .
$$

Since $k_{i j}$ and ${ }^{3} R_{i j}$ are independent of $t$, we must have that

$$
{ }^{3} R_{i j}=2 C k_{i j}, \quad C=\text { const } .
$$

In other words, $k_{i j}$ is the metric of a space with constant curvature. 
In summary, $g_{\alpha \beta}$ has the form

$$
g_{00}=-1, \quad g_{0 i}=0, \quad g_{i j}=\mathfrak{R}^{2} k_{i j}\left(y^{i}\right)
$$

where $k_{i j}$ is the metric of a space of constant curvature. The field equations of general relativity allow us to express $w$ and $p$ in terms of $\Re$ and $C$ as follows [11]:

$$
\begin{aligned}
& w=3 k^{-1} \mathfrak{Z}^{-2}\left(C+\dot{\mathfrak{R}}^{2}\right) \\
& p=-k^{-1} \mathfrak{R}^{-2}\left(2 \mathfrak{R} \ddot{\mathfrak{R}}^{+} \dot{\mathfrak{R}}^{2}+C\right) .
\end{aligned}
$$

This metric is the Robertson-Walker cosmological metric $[9,2]$.

\section{Field Equations when $\left(\varrho u^{\sigma}\right)_{; \sigma}=0$}

In this section we examine the field equations under the assumptions that the conformal tensor has zero divergence and the rest particle density is conserved. Equation (1.11), in the light of Eq. (1.13), becomes:

or :

$$
\varrho w_{, \sigma} u^{\sigma}=(w+p) \varrho,{ }_{\sigma} u^{\sigma}
$$

$$
\varrho \theta=-\varrho,{ }_{\sigma} u^{\sigma} .
$$

The vanishing of the divergence of the conformal tensor implies that Eq. (2.9) holds. That is,

$$
u_{\alpha ; \beta}=\frac{1}{3} \theta h_{\alpha \beta}+F_{,{ }_{\tau}} h_{\alpha}^{\tau} u_{\beta} .
$$

In particular, $\omega_{\alpha \beta}=0$ and $u_{\alpha}$ is proportional to the gradient of a scalar. That is, functions $\alpha$ and $\phi$ exist such that

$$
\alpha u_{\mu}=\phi_{, \mu} .
$$

We may choose our coordinates so that $\phi=x^{0}$ and such that $g_{0 i}=0[5]$. In such a coordinate system

$$
h_{i j}=g_{i j}, \quad h_{0 i}=g_{0 i}=0, \quad h_{00}=0 .
$$

The requirement that $u_{\mu}$ be a unit vector leads to the condition that

$$
g_{00}=\left(g^{00}\right)^{-1}=-\alpha^{-2} .
$$

It is a consequence of Eq. (4.3) that

where, as in Eq. (2.10),

$$
\alpha,{ }_{\sigma} h^{\sigma}{ }_{\tau}=\alpha F,{ }_{\sigma} h^{\sigma}{ }_{\tau}
$$

and we have used

$$
F=\log (w+p)
$$

$$
w_{, \sigma} h_{\mu}^{\sigma}=0 .
$$

In the coordinate system we are using $h^{i}{ }_{j}=\delta^{i}{ }_{j}$. Hence, we may integrate Eq. (4.5) to give

$$
\log \alpha=F+k\left(x^{0}\right)
$$


where $k$ is a function of $x^{0}$ alone. Thus

$$
g_{00}=-f\left(x^{0}\right)(w+p)^{-2}
$$

and we may redefine the variable $x^{0}$ so that

$$
g_{00}=-(w+p)^{-2}=-e^{-2 F} .
$$

In this coordinate system we have

$$
\begin{aligned}
& u_{\alpha}=-(w+p)^{-1} \delta^{0}{ }_{\alpha}=-e^{-F^{\prime}} \delta^{0}{ }_{\alpha} \\
& u^{\alpha}=(w+p) \delta^{\alpha}{ }_{0}=e^{F} \delta^{\alpha}{ }_{0} .
\end{aligned}
$$

We now turn to Eq. (4.2). If either $\alpha$ or $\beta$ equals zero in these equations, the equations are satisfied as a consequence of Eqs. (4.6) and (4.7). We are then left with the equations

$$
u_{i ; j}=-u_{0} \Gamma_{i j}^{0}=\frac{1}{2}(w+p) \dot{g}_{i j}=-\frac{1}{3} \dot{\varrho} \varrho^{-1}(w+p) g_{i j}
$$

where the dot denotes the derivative with respect to $x^{0}$, as follows from Eqs. (4.1) to (4.7). Hence we must have

or

$$
\varrho \dot{g}_{i j}=-\frac{2}{3} g_{i j} \dot{\varrho}
$$

$$
g_{i j}=\varrho^{-2 / 3} k_{i j}\left(x^{k}\right)
$$

where the $k_{i j}$ are functions of the variables $x^{1}, x^{2}, x^{3}$ alone.

The solution of the field equations is thus reduced to a discussion of the functions $w\left(x^{0}\right), \varrho\left(x^{0}, x^{k}\right)$ and the tensor $k_{i j}\left(x^{k}\right)$. Since the thermodynamic variables $w, \varrho$, and $p$ may be considered as functions of two such variables, we may treat all thermodynamic variables as functions of $w$, the rest energy density, and $S$, the entropy. These are convenient variables for our purposes, since in the coordinate system given above

and

$$
w_{, i}=0
$$

$$
\dot{S}=0
$$

as follows from Eqs. (1.13) and (2.3).

Hence, for any thermodynamic variable such as $\varrho$ or $p$ we have

$$
\dot{f}=f_{w} \dot{w} ; \quad f_{, i}=f_{s} S_{, i} ; \quad \dot{f}_{, i}=f_{s w} S_{, i} \dot{w}
$$

where the subscript $w$ or $S$ denotes the partial derivative of $f(w, S)$ with respect to the indicated variable.

We now turn to a discussion of the field equations. It may be verified that these equations may be written as

$$
\begin{aligned}
R_{00} & =(w+p)^{-2}\left[(w+p) \ddot{w}-\frac{1}{3} \dot{w}^{2}-\bar{F}_{i j} g^{i j}\right] \\
& =\frac{1}{2} k(w+p)^{-2}(w+3 p)
\end{aligned}
$$

and

$$
R_{i j}={ }^{3} R_{i j}+\bar{F}_{i j}-\frac{1}{3}\left[\ddot{w}(w+p)-\dot{w}^{2}\right] g_{i j}=\frac{1}{2} k(w-p) g_{i j}
$$


where ${ }^{3} R_{i j}$ is the three-dimensional Ricci tensor formed from the $g_{i j}$,

$$
\begin{aligned}
\bar{F}_{i j} & =F_{\backslash i j}-F_{, i} F,{ }_{j} \\
F & =\log (w+p)
\end{aligned}
$$

and the bar denotes the covariant derivative with respect to the metric $g_{i j}$.

We may also rewrite Eqs. (4.9) and (4.10) in terms of the metric $k_{i j}$. We obtain

$R_{00}=(w+p)^{-2}\left[(w+p) \ddot{w}-\frac{1}{3} \dot{w}^{2}\right]-$

$-(w+p)^{-2}\left[\varrho^{2 / 3} k^{i j} \widetilde{\bar{F}}_{i j}-\frac{1}{3} \varrho^{-1 / 3} k^{i j}{ }^{\prime},{ }_{j} \varrho, i\right]=\frac{1}{2} k(w+p)^{-2}(w+3 p)$

and

$$
\begin{aligned}
R_{i j} & =K_{i j}+\sigma_{\| i j}+\sigma_{, i} \sigma_{, j}-2 F_{, i} F_{, j}+A^{\prime} k_{i j} \\
& =\frac{1}{2} k(w-p) \varrho^{-2 / 3} k_{i j}
\end{aligned}
$$

where $K_{i j}$ is the Ricci tensor formed from $k_{i j}$,

$$
\begin{gathered}
\sigma=F+\frac{1}{3} \log \varrho \\
\overline{\bar{F}}_{i j}=F_{\| i j}-F,{ }_{i} F,{ }_{j} .
\end{gathered}
$$

The double bar denotes the covariant derivative with respect to $k_{i j}$, and $A^{\prime}=\frac{1}{3}\left[k^{i j} \frac{\varrho_{\| i j}}{\varrho}-\frac{4}{3} k k^{i j} \frac{\varrho_{, i}}{\varrho} \frac{\varrho_{, j}}{\varrho}-\varrho^{-2 / 3}\left(\ddot{w}(w+p)-\dot{w}^{2}\right)-\frac{\varrho_{, i}}{\varrho} F{ }_{, j} k^{i j}\right]$. We may write Eqs. (4.12) and (4.13) as, respectively:

$$
\begin{aligned}
\frac{1}{2} k(w & +3 p)=(w+p) \ddot{w}-\frac{1}{3} \dot{w}^{2}-(w+p)^{-1} \varrho^{2 / 3} p_{s} k^{i j} S_{\| i j}+ \\
& +(w+p)^{-2} \varrho^{2 / 3} k^{i j} S_{, i} S_{, j}\left[(w+p)\left(\frac{p_{s} \varrho_{s}}{3 \varrho}-p_{s s}+2 p_{s}{ }^{2}\right]\right.
\end{aligned}
$$

and

$$
K_{i j}=A k_{i j}-B S_{\| i j}+C S_{, i} S_{, j} .
$$

The quantities $A, B$, and $C$ are

$$
\begin{gathered}
A=\frac{1}{2} k(w-p) \varrho^{-2 / 3}-A^{\prime} \\
=\varrho^{-2 / 3}\left[\frac{1}{3} \ddot{w}(w+p)-\frac{1}{3} \dot{w}^{2}+\frac{1}{2} k(w-p)\right]-\frac{1}{3} S_{\| i j} k^{i j} \varrho^{-1} \varrho_{s}+ \\
+\frac{1}{3} \varrho^{-1} S, i,{ }_{, j} k^{i \jmath}\left(-\varrho_{s s}+\frac{4}{3} \varrho_{s}^{2} \varrho^{-1}+(w+p)^{-1} \varrho_{s} p_{s}\right) \\
B=p_{s}(w+p)^{-1}+\frac{1}{3} \varrho_{s} \varrho^{-1}=\left[\log \varrho^{1 / 3}(w+p)\right]_{s} \\
C=-\frac{1}{3} \varrho^{-1} p_{s s}-(w+p)^{-1} p_{s s}+\frac{2}{9} \varrho^{-2} \varrho_{s}{ }^{2}+ \\
+2(w+p)^{-2} p_{s}{ }^{2}-\frac{2}{3} \varrho^{-1}(w+p)^{-1} \varrho_{s} p_{s}
\end{gathered}
$$


The tensors $K_{i j}$ and $k_{i j}$ and the function $S$ which enter into Eqs. (4.15) depend only on the variables $x^{i}(i=1,2,3)$. Hence the coefficients $A, B$, and $C$ must be restricted in their dependence on $x^{0}$. We shall now examine the nature of this restriction. We observe that by using Eq. (4.14) to eliminate $\ddot{w}$, the quantity $A$ may be expressed as a function of $\dot{w}$, the thermodynamic variables $w$ and $S$, and the quantities

and

$$
\Delta_{2} S=k^{i j} S_{\| i j}
$$

$$
\Delta_{1} S=k^{i j} S_{i} S_{, j}
$$

which are functions of $x^{i}(i=1,2,3)$ alone. We have

$$
\begin{aligned}
A= & \frac{2}{3} \varrho^{-2 / 3}\left(k w-\frac{1}{3} \dot{w}^{2}\right)+\frac{1}{3} \Delta_{2} S \Delta_{1} S\left(\frac{p_{s}}{w+p}-\frac{\varrho_{s}}{\varrho}\right)+ \\
& +\frac{1}{3} \Delta_{1} S\left(-\frac{\varrho_{s s}}{\varrho}+\frac{4}{3} \frac{\varrho_{s}^{2}}{\varrho^{2}}+\frac{2}{3} \frac{\varrho_{s}}{\varrho} \frac{p_{s}}{(w+p)}+\frac{p_{s s}}{(w+p)}-\frac{2 p_{s}{ }^{2}}{(w+p)^{2}}\right)
\end{aligned}
$$

Hence we may write

$$
\dot{A}=\dot{w} \hat{A}
$$

where $\hat{A}$ is a function of the same quantities that enter in $A$.

When Eq. (4.15) is differentiated with respect to $x^{0}$ we obtain

$$
A k_{i j}-\dot{B} S_{\| i j}+\dot{C} S_{, i} S_{, j}=0
$$

since $k_{i j}$ and hence $K_{i j}$ are independent of $x^{0}$. This equation may in turn be written as

$$
\dot{w}\left(\hat{A} k_{i j}-B_{w} S_{\| i j}+C_{w} S,{ }_{i} S,{ }_{j}\right)=0
$$

since $B$ and $C$ are formed from matter variables.

It follows from Eq. (4.19) that either

$$
\dot{w}=0
$$

and hence the metric tensor $g_{\mu \nu}$ is static, or else

$$
B_{w} S_{\| i j}=\hat{A} k_{i \jmath}+C_{w} S_{, i} S_{, j} .
$$

We shall treat the static case in the next section. We now assume that Eq. (4.21) holds. Then if

we must also have

$$
B_{w}=0
$$

$$
C_{w}=\hat{A}=0
$$

since $k_{i j}$ must be non-singular. Now we may write

$$
C=-B_{s}-B^{2}+2 p_{s}^{2}(w+p)^{-2} .
$$

Equation (4.22) implies that

$$
\varrho^{1 / 3}(w+p)=f(S) g(w) .
$$


The first of Eqs. (4.23) and (4.24) imply that

hence

$$
w+p=f^{*}(S) g^{*}(w)
$$

$$
\varrho=f * *(S) g * *(w) .
$$

It then follows from the expression given above for $A$ that we must have

$$
k w-\frac{1}{3} \dot{w}^{2}=\alpha g^{* *}(w)^{2 / 3}
$$

where $\alpha$ is a constant. Thus the dependence of $w$ on $t$ may be determined once the function $g^{* *}(w)$ is known.

If the caloric equation of state of the fluid is such that Eqs. (4.25) and (4.26) do not hold, $B_{w} \neq 0$, and we may write Eq. (4.21) as

$$
S_{\| i j}=B_{w}{ }^{-1} \hat{A} k_{i j}+B_{w}{ }^{-1} C_{w} S_{, i} S_{, j}
$$

and Eq. (4.15) becomes

$$
K_{i j}=\left(A-B B_{w}{ }^{-1} \hat{A}\right) k_{i j}-\left(B B_{w}{ }^{-1} C_{w}-C\right) S_{, i} S_{, j} .
$$

Again we may differentiate Eq. (4.28) with respect to $x^{0}$ and find that the coefficients of $k_{i j}$ and $S_{, i} S_{, j}$ must be functions of $x^{i}$ alone. In particular we must have

Hence

$$
B B_{w}{ }^{-1} C_{w}-C=k(S) .
$$

$$
C=k(S)+\ell(S) B
$$

where $k$ and $\ell$ may be arbitrary functions of the entropy $S$. In view of Eq. (4.24), Eq. (4.29) may be regarded as a differential condition on $B$ and $(w+p)$.

Equation (4.27) then becomes

where

$$
S_{\| i j}=\alpha(x) k_{i j}+\ell S_{, i} S_{, j}
$$

$$
\hat{A}=\alpha B_{w}
$$

and $\alpha$ is a function of $x^{i}(i=1,2,3)$ alone. The latter equation may be regarded as a first order differential equation for $w$ as a function of $x^{0}$ since it contains $\dot{w}$ and terms which depend on thermodynamic variables as well as terms involving $\Delta_{2} S$ and $\Delta_{1} S$.

If the caloric equation of state of the fluid is such that Eq. (4.29) is satisfied, Eq. (4.28) becomes

$$
K_{i j}=(A-B \alpha) k_{i j}-k(S) S_{, i} S_{, j} .
$$

In order that Eqs. (4.30) and (4.32) admit solutions for the $k_{i j}$ and the function $S$, the integrability conditions of Eq. (4.30) must be satisfied. These come from [12]

$$
S_{\| i[j k]}=\frac{1}{2} S_{, t} K^{t}{ }_{i j k}=S_{,{ }_{[j}} K_{k] i}+S_{, t} K^{t}{ }_{[j} k_{k] i}+\frac{1}{2} K S_{,[k} k_{j] i}
$$


since the curvature tensor of a three-space is expressible in terms of the Ricci tensor and the scalar curvature of such a space [5]. If we substitute from Eq. (4.32) into this equation we obtain

$$
S_{\bigcap_{i j k]}}=\frac{1}{2}\left(A-B \alpha-k \Delta_{1} S\right) S,{ }_{[j} k_{k] i} .
$$

When one computes the left hand side of this equation from Eq. (4.30) one obtains the equation

$$
\left[\left(\alpha,\left[j-\ell \alpha S,{ }_{[j}\right)+\frac{1}{2}\left(A-B \alpha-k \Delta_{1} S\right) S_{,[j]}\right] k_{k] i}=0 .\right.
$$

Hence we must have

$$
\alpha_{, j}=-\left[\frac{1}{2}(A-B \alpha)-\frac{1}{2} k \Delta_{1} S-\ell \alpha\right] S_{, j}=D S_{, j} .
$$

Then it follows that the coefficient of $S,{ }_{j}$ must be a function of $S$ alone and hence $\alpha$ must be a function of $S$. Equations (4.30) and (4.31) may be used to determine $\Delta_{2} S$ as a function of $\Delta_{1} S$ and thermodynamic variables after $w\left(x^{0}\right)$ is determined. The quantity $\Delta_{1} S$ may be determined as a function of the thermodynamic variables alone from the requirement that $D$ is a function of $S$.

Thus we see that $\dot{w} \neq 0$ and $S,{ }_{i} \neq 0$ can obtain only if there are restrictions on the kind of matter present. The case for which $B_{w}=\hat{A}$ $=C_{w}=0$ is similar to the static case $\dot{w}=0$ which will be discussed in the next section. In case $B_{w} \neq 0$ and a general caloric equation of state holds, so that Eq. (4.29) does not obtain, we must have

$$
S_{, i}=0 \text {. }
$$

That is, $S$ is a constant. Hence the fluid obeys an equation of state of the type discussed previously, and the metric tensor of space-time is of the Robertson-Walker type.

We shall not discuss the restricted fluids any further but shall turn to a discussion of the static case.

\section{The Static Case}

We have seen that this case is characterized by the condition

$$
\dot{w}=0
$$

for in this case all thermodynamic variables are independent of $x^{0}$ : They depend on $x^{0}$ only insofar as they depend on $w$. Hence, $w+p$ and $\varrho$ are independent of $x^{0}$ and it follows from our previous work that $\dot{g}_{\mu \nu}=0$ in the coordinate system used above.

It is most convenient to work with the field equations in the form given by Eqs. (4.9) and (4.10). These equations become

$$
\frac{1}{2} k(w+3 p)=-\left(F_{\mid i j}-F_{, i} F_{, j}\right) g^{i j}
$$


and

$$
\frac{1}{2} k(w-p) g_{i j}={ }^{3} R_{i j}+F_{\mid i j}-F_{, i} F_{, j}
$$

where the bar denotes the covariant derivative with respect to the $y_{i j}$ and

$$
F=\log (w+p) .
$$

It follows from these equations that

and

$$
{ }^{3} R_{i j}=-\left(F_{\mid i j}-F,{ }_{i}{ }_{, j}\right)-g_{i j}\left[g^{s t}\left(F_{\mid s t}-F_{, s} F_{, t}\right)+2 k p\right]
$$

$$
{ }^{3} R=-4 g^{i j}\left(F_{\mid i j}-F_{, i} F_{, j}\right)-6 k p=2 k w .
$$

Since $w=$ constant, the scalar curvature ${ }^{3} R$ is constant.

It follows from Eq. (5.4) alone that the contracted Bianchi identities become

$$
\left({ }^{3} R^{i}{ }_{j}-\frac{1}{2} \delta^{i}{ }_{j}{ }^{3} R\right)_{\mid i}=F_{, j}\left[k(w+3 p)+2 g^{s t}\left(F_{\mid s t}-F_{, s} F_{, t}\right)\right]=0 .
$$

Hence Eqs. (5.4) imply (5.1) and (5.2) when $F_{, j} \neq 0$. The case $F_{,_{j}}=0$ corresponds to the Einstein universe, a special Robertson-Walker metric [13].

Equations (5.4) may be written as

$$
{ }^{3} R_{i j}-\frac{1}{4}{ }^{3} R g_{i j}=-\left(F_{1 i j}-F,{ }_{i} F,{ }_{j}\right)-\frac{1}{2} k p g_{i j} .
$$

Look on Eq. (5.6) as a differential equation for $F$. The integrability conditions for the function $F$ are

$$
\begin{aligned}
& \left({ }^{3} R_{i[j}-\frac{1}{4}{ }^{3} R g_{i[j}\right)_{\mid k]}={ }^{3} R_{i[j \mid k]} \\
& =-F{ }_{s}\left[2 \delta^{s_{[j}}{ }^{3} R_{k] i}+{ }^{3} R^{s}{ }_{[j} g_{k] i}+{ }^{3} R \delta_{[k}^{s_{[k}} g_{j] i}\right]
\end{aligned}
$$

since ${ }^{3} R$ is a constant.

The nine Eqs. (5.7) contain only five equations independent of the condition

for they may be written as

$$
{ }^{3} R^{i}{ }_{j \mid i}=0
$$

where

$$
Z_{i j}=Z_{j i} \equiv{ }^{3} R_{i k \mid m} E^{k m_{j}}=2 F,{ }_{m}^{3} R_{k\left({ }_{i} E_{j)}{ }^{k m}\right.}
$$

$$
E^{i j k}=g^{-\frac{1}{2}} \varepsilon^{i j k}
$$

and $\varepsilon^{i j k}$ is the completely antisymmetric tensor density with $\varepsilon^{123}=1$; $g$ is the determinant of $g_{i j}$. We must also have

$$
g^{i j} Z_{i j}=0
$$

as a consequence of the symmetry of ${ }^{3} R_{i j}$. That $Z_{i j}$ is symmetric follows from Eq. (5.8). 
Thus, in order that Eq. (5.9) admit a solution for $F,{ }_{m}$ we must in general have two equations satisfied by the coefficients of $F, m$ in these equations and the $Z_{i j}$. These equations may be written as

and

$$
{ }^{3} R^{i j} Z_{i j}={ }^{3} R^{i j 3} R_{i k \mid m} E^{k m}{ }_{j}=0
$$

$$
{ }^{3} R_{s}^{i}{ }^{3} R^{s j} Z_{i j}={ }^{3} R_{s}^{i}{ }^{3} R^{s j}{ }^{3} R_{i k \mid m} E^{k m_{j}}=0 .
$$

The remaining three equations in Eqs. (5.9) may be written as

where

$$
A_{u}=-F,{ }_{s} S^{s}{ }_{u}
$$

and

$$
A_{u}=\left({ }^{3} R_{i u \mid j}-{ }^{3} R_{i j \mid u}\right)^{3} R^{i j}
$$

$$
S^{u}{ }_{v}=\left({ }^{3} R^{2}-2^{3} R^{s}{ }_{t}^{3} R^{t}\right) \delta^{u}{ }_{v}+3{ }^{3} R^{u}{ }_{s}{ }^{3} R^{s}{ }_{v}-2^{3} R^{u}{ }_{v}{ }^{3} R .
$$

Equations (5.11), (5.12), and (5.13) are equivalent to Eqs. (5.9). From Eq. (5.13) it follows that if $A_{k}=0$ the rank of $S^{u}{ }_{v}$ must be less than three. It may be shown that this implies that ${ }^{3} R^{i}{ }_{j}$ have at least two equal proper values; that is, that the three-space with the metric $g_{i j}$ have two equal principal curvatures. Note that if $g_{i j}$ is the metric of a space of constant curvature, so that ${ }^{3} R^{i}{ }_{j}$ has three equal proper values, then Eq. (5.9) is satisfied for arbitrary $F_{, i}$.

The three-space with metric tensor $g_{i j}$ must therefore have (a) a constant scalar curvature and (b) a Ricci tensor which has zero divergence and which satisfies Eqs. (5.11), (5.12), and (5.13) in order that it be the space $x^{0}=$ constant in a static space-time with vanishing divergence of the conformal tensor with a fluid present. We shall not discuss these three-spaces further, but will consider the special case for which the static space-time is conformally flat.

It then follows from Eq. (2.20) that

where

$$
F_{Q \tau} h_{\alpha}{ }_{\alpha} h_{\beta}^{\tau}=\frac{1}{3} F_{\varrho \tau} h^{\rho \tau} h_{\alpha \beta}
$$

$$
F_{\sigma \tau}=F_{; \sigma \tau}-F_{, \sigma} F_{, \tau}
$$

and the semi-colon denotes the covariant derivative with respect to $g_{\mu \nu}$. In view of the fact that $F,_{0}=0$ and the line element is static, Eq. (5.14) becomes

$$
F_{\mid i j}-F_{, i} F_{, j}=\frac{1}{3}\left(F_{\mid s t}-F,{ }_{s} F,{ }_{t}\right) g^{s t} g_{i j} .
$$

Equations (5.1) and (5.2), the field equations, become

and

$$
F_{\mid i j}-F{ }_{i} F_{, j}=-\frac{1}{6} k(w+3 p) g_{i j}
$$

$$
{ }^{3} R_{i j}=\frac{2}{3} k w g_{i j}
$$

18 Commun. math. Phys., Vol. 5 
Thus the three-space with metric tensor $g_{i j}$ is a space of constant curvature. In this case, Eqs. (5.9), the integrability conditions for Eq. (5.15), are identically satisfied. That is, Eqs. (5.9) introduce no restrictions on the $F,{ }_{m}$.

Since

$$
F=\log (w+p)
$$

we may write Eq. (5.15) as

where

$$
Y_{\mid i j}=\frac{1}{6} k(3-2 w Y) g_{i j}
$$

$$
Y=e^{-F}=(w+p)^{-1} .
$$

In order to solve Eq. (5.17) for $Y$, we introduce a coordinate system $x^{1}=r, x^{2}=\theta, x^{3}=\phi$ in the three-space such that

where

$$
\begin{aligned}
& g_{11}=R^{2}\left(R^{2}-r^{2}\right)^{-1}, \quad g_{12}=g_{13}=g_{23}=0, \\
& g_{22}=r^{2}, \quad \text { and } g_{33}=r^{2} \sin ^{2} \theta
\end{aligned}
$$

$$
k w R^{2}=3 .
$$

The solution of Eq. (5.17) is straightforward. The result is that $Y$ is a function only of $r$ and is given by:

$$
Y=\frac{1}{2} k R^{2}-A\left(R^{2}-r^{2}\right)^{\frac{1}{2}}
$$

where $A$ is a constant. The four-dimensional metric is then given by Eq. (5.19) and

$$
\begin{aligned}
& g_{0 i}=0 \quad(i=1,2,3) \\
& g_{00}=Y^{2}=\left[\frac{1}{2} k R^{2}-\left(A R^{2}-r^{2}\right)^{\frac{1}{2}}\right]^{2} .
\end{aligned}
$$

This is the form that Tolman gives for the Schwarzschild interior solution [13].

If the constant $A$ is taken to be zero, we have

$$
w+3 p=0
$$

and the solution given above reduces to the Einstein universe [13].

\section{Discussion and Conclusion}

Modern methods of treating gravitational radiation rely heavily on algebraic statements concerning the conformal tensor. In a vacuum region, a conformal tensor satisfying an algebraic condition

$$
f\left(C_{\gamma \delta}^{\alpha \beta}, g_{\mu \nu}\right)=0
$$

has many properties in analogy with electromagnetic radiation [14]. For example, a pure radiation field is expected to obey

$$
C^{\alpha \beta_{\sigma \tau}} C^{\sigma \tau}{ }_{\gamma \delta}=0
$$

which is characteristic of a Petrov type Null space-time. 
The final identification of algebraically special conformal tensors with gravitational radiation has not been made, however, since the effect of matter on algebraic type is not well known. With this qualification in mind, the following very interesting question may none-the-less be asked: Which algebraic types of conformal tensor are compatible with which types of stress-energy tensor? In other words, if a conformal tensor is desired which satisfies an equation such as Eq. (6.1), is it possible at the same time to find a $T_{\mu \nu}$ which satisfies some equation such as

$$
h\left(T_{\alpha \beta}, g_{\mu \nu}\right)=0 \text { ? }
$$

Since $T_{\mu \nu}$ and $C^{\alpha \beta}{ }_{\gamma \delta}$ are algebraically distinct, there is no difficulty in satifying both Eqs. (6.1) and (6.2) at a point. The Bianchi identities, however, link these two tensors by a differential relation. Hence there is difficulty in satisfying algebraic conditions on the stress-energy tensor and the conformal tensor over a region as may be seen from a theorem due to Szekreres [8]: A type Null conformal tensor (non-zero) is impossible in a dust-filled model. Note that a dust-filled model is equivalent to the algebraic statement [3]

where $\varrho$ is positive.

$$
T^{\mu}{ }_{\sigma}\left(T^{\sigma}{ }_{\nu}+\varrho \delta^{\sigma}{ }_{\nu}\right)=0
$$

The theorems in this paper show that, in contrast to the type Null case, a zero conformal tensor is compatible with a fluid. A zero conformal tensor, however, greatly restricts the dependence of the fluid variables on spatial and on time variables.

In fact, we started not with the vanishing of $C^{\alpha \beta}{ }_{\gamma \delta}$, but with the vanishing of the conformal divergence:

$$
C_{\gamma \delta ; \sigma}^{\alpha \sigma}=0 \text {. }
$$

That Eq. (6.4) restricts the stress-energy tensor has been shown before [15]. Here we have shown that Eq. (6.4) in the presence of a perfect fluid satisfying either an equation of state or conservation of mass requires either that the space-time be static or that it be a RobertsonWalker metric - or that the special thermodynamic relations discussed in Section 4 hold.

If, in addition to Eq. (6.4), the space-time is conformally flat, then, in the absence of the special caloric relations of Section 4, there are two cases: First, the non-static case consists of the Robertson-Walker solutions. Second, the static case consists of the Schwarzschild interior solutions. Other conformally flat models, in which the conservation of rest particle density does not necessarily hold, are being studied by Plebański [16].

In conclusion, let us repeat our postulates. (1) The algebraic postulate of vanishing conformal divergence, Eq. (6.4), was made. (2) We postulated $18^{*}$ 
that the material in the model be a perfect fluid, an algebraic postulate. (3) We postulated that either (3a) an equation of state, $p=p(w)$, hold, or else $(3 \mathrm{~b})$ the rest particle density $\varrho$ be conserved, $\left(\varrho u^{\sigma}\right)_{; \sigma}=0$.

The postulates were used to give the form of the metric coefficients. If case (3a) holds, or if case $(3 \mathrm{~b})$ is supplemented with the postulate of conformal flatness, it was shown that the results are models more familiarly derived from isotropy postulates. These models are the Robertson-Walker metrics and the Schwarzschild interior solutions respectively.

\section{References}

1. Hawking, S. W.: Astrophys. J. 145, 544 (1966).

2. Walker, A. G.: Quart. J. Math. Oxford Ser. 6, 81 (1935).

3. TAUB, A. H.: Arch. Ratl. Mech. Anal. 3, 312 (1959). Also "Relativistic hydrodynamics", notes.

4. Ehlers, J.: Akad. Wiss. Mainz Abh. Math.-Naturwiss. (1961) No. 11.

5. Eisenhart, L. P.: Riemannian geometry. Princeton: Princeton Univ. Press 1964.

6. TaUb, A. H.: In: Perspectives in geometry and relativity (ed. by B. Hoffman). Bloomington: Indiana Univ. Press 1966.

7. Kundt, W., and M. TrüMPer: Akad. Wiss. Mainz Abhdl. Math.-Naturwiss. (1962) No. 12.

8. Szekeres, P.: J. Math. Phys. 7, 751 (1966).

9. Robertson, H. P.: Rev. Mod. Phys. 5, 62 (1933).

10. Raychaudhuri, A.: Phys. Rev. 98, 1123 (1955); 106, 172 (1957).

11. McVittre, G. C.: General relativity and cosmology. London: Chapman and Hall 1956.

12. Schouten, J. A.: Ricci-Calculus. Berlin-Göttingen-Heidelberg: Springer 1954.

13. Tolman, R. C.: Relativity thermodynamics and cosmology. Oxford: Clarendon Press 1962.

14. Pirani, F. A. E.: In: Gravitation, an introduction to current research (ed. by L. Witten). New York: Wiley 1962.

15. Szekeres, P.: Proc. Roy. Soc. London A 274, 206 (1963).

16. Plebax́ski, J.: Private communication. 